\title{
Antireflective Huygens' Metasurface with Correlated Disorder Made from High-Index Disks Implemented into Silicon Heterojunction Solar Cells
}

\author{
Peter M. Piechulla, ${ }^{*}{ }^{\#}$ Evgeniia Slivina, ${ }^{*}, \#$ Derk Bätzner, Ivan Fernandez-Corbaton, Prerak Dhawan, \\ Ralf B. Wehrspohn, Alexander N. Sprafke, and Carsten Rockstuhl
}

Cite This: https://doi.org/10.1021/acsphotonics.1c00601

Read Online

ABSTRACT: A large variety of different strategies has been proposed as alternatives to random textures to improve light coupling into solar cells. While the understanding of dedicated nanophotonic systems deepens continuously, only a few of the proposed designs are industrially accepted due to a lack of scalability. In this Article, a tailored disordered arrangement of high-index dielectric submicron-sized titanium dioxide $\left(\mathrm{TiO}_{2}\right)$ disks is experimentally exploited as an antireflective Huygens' metasurface for standard heterojunction silicon solar cells. The disordered array is fabricated using a scalable bottom-up technique based on colloidal self-assembly that is applicable virtually irrespective of material or surface morphology of the device. We observe a broadband reduction of reflectance resulting in a relative improvement of a short-circuit current by $5.1 \%$ compared to a reference cell with an optimized flat antireflective indium tin oxide (ITO) layer. A theoretical model based on Born's first approximation is proposed that links the current increase in the arrangement of disks expressed in terms of the structure factor $S(q)$ of the disk array. Additionally, we discuss the optical performance of the metasurface within the framework of helicity preservation, which can be achieved at specific wavelengths for an isolated disk for illumination along the symmetry axis by tuning its dimensions. By comparison to a simulated periodic metasurface, we show that this framework is applicable in the case of the structure factor approaching zero and the disks' arrangement becoming stealthy hyperuniform.

KEYWORDS: scalable, hyperuniformity, tailored disorder, Mie resonators, Kerker condition, metamaterials

$\mathrm{E}$ fficient light coupling between a device and its surrounding is key to maximize the performance of any optoelectronic system. This is particularly true for silicon solar cells, where the large discontinuity of the permittivity from air to silicon causes severe reflection losses. Optical losses are indeed one of the main causes that prevents us currently from reaching the detailed-balance limit, ${ }^{1,2}$ which constitutes the upper bound of achievable device efficiency.

Common strategies to reduce losses include the use of flat antireflective coatings and surface textures, or combinations thereof, for example, pyramidal textures with an added conformal coating. The latter approach has been overwhelmingly successful as industry standard for decades, albeit it may not be applicable for every device. For example, it requires comparably thick wafers, while thin absorbers are attractive for flexible devices and to lower material consumption. Also, it relies on silicon as the absorber material because the pyramids are etched in a self-organized process along specific crystallographic axes. The approach is not applicable to promising alternative materials, such as perovskites, that may soon be industrialized. ${ }^{3,4}$ Silicon pyramids imply a corrugated interface, while very often a flat interface is preferred in the following fabrication steps. For example, in solution-processed perovskite-silicon tandem solar cells, the pyramidal surface profile poses additional challenges in optimizing the electrical functionality of the perovskite topcell. $^{5-7}$ Alternatives to pyramidal textures must, therefore, be explored, that come with greater flexibility with respect to the design of a device. If, for instance, the tandem is bound to a flat configuration, a light in-coupling structure could be added on top of the perovskite top cell.

That challenge has been accepted, and a number of nanophotonic concepts have been proposed not only to improve light incoupling on the front interface, but for a much broader spectrum of objectives, including light trapping, spectrum splitting in tandem devices, or colored photovoltaics. $^{8-12}$ As building blocks, submicron-sized disks or pillar-shaped scattering elements have attracted a lot of attention during recent years because of their ability to support multipolar Mie resonances. ${ }^{13,14}$ This allows to control

Received: April 20, 2021 
the propagation of light in quite a drastic and deterministic manner. The shape of such submicron-sized disks (henceforth referred to as disks) can be tuned to carefully overlap electric and magnetic modes to control scattering characteristics. ${ }^{15-18}$ Inspired by the original work of Kerker et al., complete suppression of back scattering can be achieved, making these disk-based Huygens' metasurfaces ideal for use as antireflective coatings. ${ }^{19-21}$ This bears the question of how to arrange the scattering elements. Disordered arrangements have been shown to possess a superior broadband response, ${ }^{22-25}$ whereas periodic arrangements can be made entirely subwavelength, thereby suppressing all off-axis scattering. ${ }^{19}$ As we will show, the latter can be achieved also in a disordered arrangement if spatial correlations are tuned appropriately. ${ }^{26,27}$

The design of the metasurface proposed in this Article was inspired by our previous work ${ }^{28}$ on a similar but periodic arrangement of disks, whose antireflective properties were designed and analyzed from the perspective of the helicity of the scattered light. Preserving the helicity of the incident light, plus a sufficiently high degree of rotational symmetry along the illumination axis, are sufficient conditions to ensure zero specular reflection from a general system. ${ }^{29}$ The most general way to achieve helicity preservation is by means of electromagnetically dual systems, that is, systems with equivalent electric and magnetic responses. ${ }^{30}$ In a more restricted and practical setting, helicity preservation can be achieved for onaxis illumination of high-permittivity disks. The aspect ratio of the disks can be tuned into the Huygens scattering regime where the electric and magnetic dipolar responses are balanced. Then, the condition of discrete rotational symmetry $2 \pi / n$, with $n \geq 3$, can be met by arranging the disks in a square or hexagonal lattice on top of a substrate. While this approach nulls the specular reflection at a single or at most at a few discrete wavelengths, it turns out that the effect is all together rather broadband and suitable to the relevant spectral range for a solar cell. ${ }^{28}$ In a disordered system, the concept of discrete rotational symmetry is not exactly applicable. However, if the pattern is tailored appropriately, one can speak of an effective continuous rotational symmetry $(n \rightarrow \infty)$ and, consequently, establish a one-to-one relationship in the long wavelength limit between the helicity preservation and the antireflective properties of the coated solar cell.

Despite the initially discussed advantages of photonic structures, the proposed designs were barely even considered to be implemented on an industrial scale. On the one hand, wafer-based silicon solar cells are still dominant and the standard pyramidal texture already poses a tough benchmark. On the other hand, fabrication of newly designed structures often relies on expensive top down fabrication methods such as e-beam lithography. Also, many fabrication techniques require specially prepared substrates, for example, polished surfaces. Clearly, scalability is essential for a structure to be used in a real world application. Techniques like nanoimprint lithography $^{31,32}$ are in principle scaleable, and the first promising results were published for solar cells in the subcentimeter range. $^{33}$ However, to the best of our knowledge, so far no results for disk-based photonic structures were published for solar cells with multiple square centimeter area.

In this publication, we will show for the first time by means of experiments and simulations that a dielectric disk-based antireflective coating (Figure 1) can improve the performance of a solar cell of standard industrial design, where the point of departure is a nontextured, damage-etched surface. We

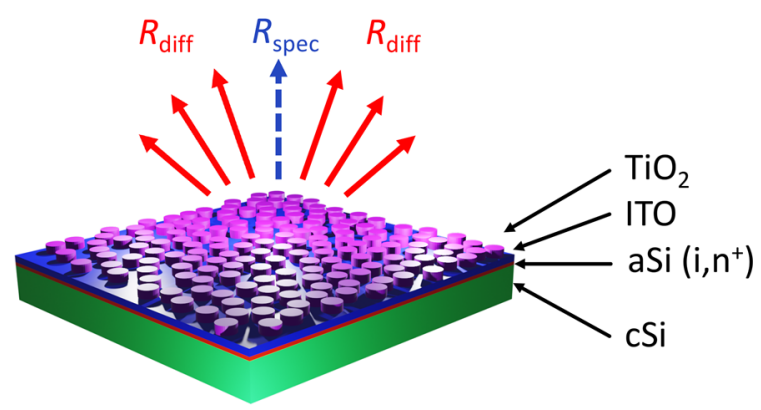

Figure 1. Schematic illustration of the solar cell structure considered in this work. $R_{\text {diff }}$ and $R_{\text {spec }}$ denote the diffuse and specular parts of reflectance as discussed in the text below. The disks were deposited on fully processed heterojunction technology $(\mathrm{HJT})$ rear-emitter solar cells for which the front surface of a flat unpolished silicon wafer was passivated by amorphous silicon (aSi) intrinsic and $n^{+}$-doped layers. A transparent conductive indium tin oxide (ITO) film serves as both an antireflection coating (ARC) and a front contact (see Methods for more details). Additionally, devices included screen-printed contact fingers (not shown) but no busbar.

consider silicon solar cells as a platform to demonstrate feasibility. However, we note that through our fabrication process the disks can be applied to any surface, in principle irrespective of topography or material. Moreover, a structure composed of dielectric disks can potentially be integrated into the solar module, which was numerically shown in ref 34 . The design of the disks is inspired by the concept of helicity preservation which, as we will show, also works in conjunction with a disk pattern of correlated disorder in the long wavelength limit. Differences to a similar but periodic system are revealed by supporting simulations thereof.

\section{RESULTS AND DISCUSSION}

Details of our novel colloid-based nanofabrication method are documented in Methods and in previous publications. ${ }^{18,35} \mathrm{We}$ fabricated arrays of disks on top of the ITO front contact of flat heterojunction solar cells, as shown in Figure 2. Like-sized disks of $370 \mathrm{~nm}$ in diameter and $122 \mathrm{~nm}$ in height (Figure 2, left) cover the entire surface of the sample in a disordered dense pattern (Figure 2, center). The process is not affected by any previously present structures (see Supporting Information, Figure S1, for disk patterns on pyramids) and covers the entire $39 \times 39 \mathrm{~mm}^{2}$ sample. This includes areas of uneven surface profile due to residue roughness after damage removal etch and contact fingers (see photograph, Figure 2, right). Some edge effects are visible, which are likely due to residues from lasercutting the samples from full scale industrial type solar cells, as the sample size was limited due to laboratory scale fabrication equipment for atomic layer desposition (ALD) for titanium dioxide $\left(\mathrm{TiO}_{2}\right)$ film deposition and reactive ion etching (RIE) for thin film structuring. We note that the relatively thick $\mathrm{TiO}_{2}$ layer and therefore slow ALD deposition could also be achieved by faster means such as plasma-enhanced chemical vapor deposition ${ }^{36,37}$ (PECVD) or sol-gel ${ }^{33}$ deposition. Furthermore, directional plasma etching processes are known to work in high-throughput inline machines. ${ }^{38,39}$ The pattern formation process is based on self-organization of colloids and can be realized with very basic equipment. We fabricated multiple samples coated with disk arrays of varying densities and further analyzed their optical and electrical properties by measuring broadband reflectance, current-voltage characteristic, and quantum efficiency (see Methods for more details). 

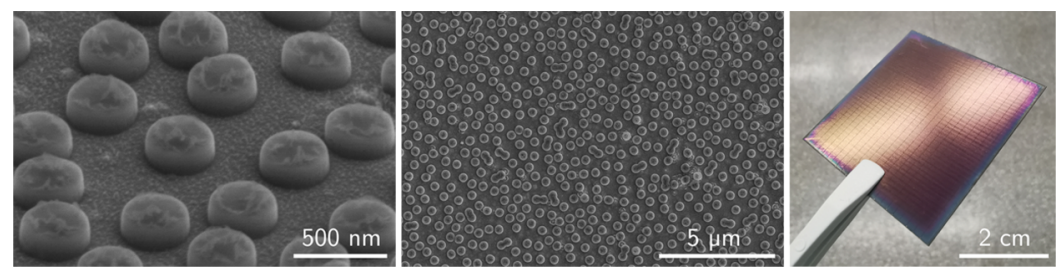

Figure 2. (Left, Center) Electron micrographs of disks deposited on top of a solar cell on different magnification stages. The left figure highlights the features of the individual disks, while the center image highlights the uniformity of the samples. (Right) Photograph of a $39 \times 39 \mathrm{~mm}^{2}$ coated solar cell.

To simulate the optical response, the disordered nature of our disk pattern is accommodated by using Born's first approximation. Far-field scattering contributions of the individual disks are conveniently superposed by a multiplication of the structure factor as an array property with the form factor squared as a property of the individual disk. Though this approach neglects any near-field interaction and multiple scattering effects, it allows reasonable predictions of the response for the presented case and will prove a useful tool of analysis. In this work, we obtain the structure factor from digitized scanning electron microscopy (SEM) images, whereas the form factor is the mere result of a simulation of a single disk by means of the finite element method (FEM; see Methods). For all simulations in this publication, we only consider reflection and scattering at the front side of the device and assume the silicon absorber to occupy the lower half-space.

In Figure 3 we use the described approach to predict the reflectance of a disk pattern deposited on top of an actual solar

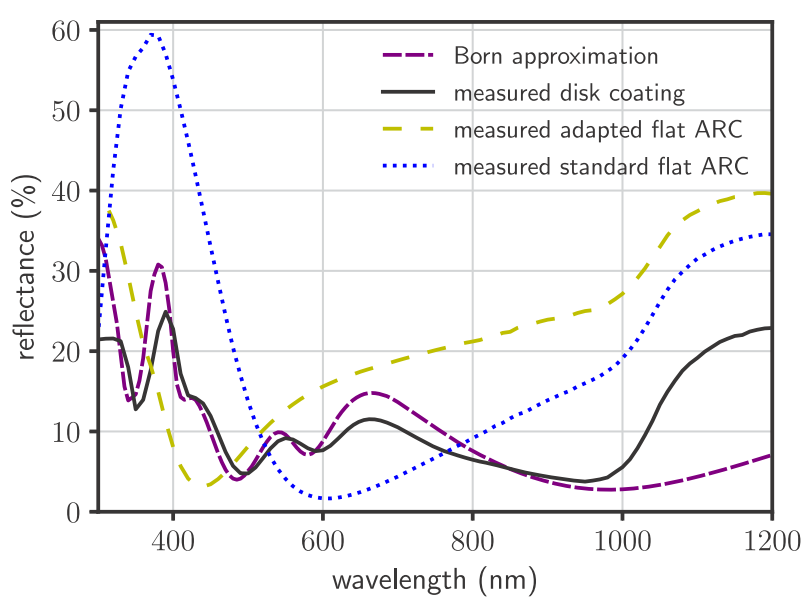

Figure 3. Reflectance of a disk pattern calculated via Born approximation and finite element simulation of a single disk. Measured reflectances of a disk coated sample and an adapted flat ARC ( $50 \mathrm{~nm}$ ITO) the disk structure is based on. A standard flat ARC reference $(80 \mathrm{~nm}$ ITO) is shown for comparison.

cell. The measured disk sample reflectance curve (see Methods for details on measurements) includes all major features predicted by the calculation, but shows an increased reflectance above $1000 \mathrm{~nm}$. This is caused by reflectance at the back side of the solar cell that has not been considered in the simulation. A detailed analysis of the measured absorption in the wavelength range around $1000 \mathrm{~nm}$ could help to assess the light-trapping capabilities of our disk structure. However, the structure was not optimized for light trapping and, given the relatively thick wafers, the experimental setup is not well suited for such an investigation. We, therefore, refer to the recent work of Slivina et al. for a detailed numerical analyses for similar but periodic light-trapping structures. ${ }^{34}$ Otherwise, the most prominent deviation of about $4 \%$ abs is found around $650 \mathrm{~nm}$ and may be the result of slight differences between the model parameters of the FEM simulation and the real disks. For instance, the surface roughness on top of the disks (Figure 2 , left) is not included in the simulation. Also, for the Born approximation, the disks are assumed to be perfectly aligned within one plane. This is in contrast to the experiment that is not conducted with polished silicon wafers.

The ITO front contact of the disk-coated solar cells has been adapted to $50 \mathrm{~nm}$ to enable good functionality of the disk structure as ARC, compared to a standard flat ARC reference with $80 \mathrm{~nm}$ ITO, that is optimized for maximum current at air mass 1.5 global tilted irradiance $\left(\mathrm{AM} 1.5 \mathrm{G}^{40}\right){ }^{28}$ Measured reflectances for both flat ARCs are shown for reference in Figure 3 as well. For the $50 \mathrm{~nm}$ ARC, the minimum in reflectance is shifted to a shorter wavelength compared to the $80 \mathrm{~nm}$ ARC. However, adding the disk coating reduces the reflectance also at longer wavelengths. Though the standard $80 \mathrm{~nm}$ ARC reflectance is lower at the optimal wavelength around $600 \mathrm{~nm}$, throughout most of the spectrum, we observe lower reflectances for the measured disk sample, thereby clearly showing the effect of the disk structure. We note that, according to our previous work, ${ }^{28}$ this result is expected to be robust to small variations of the disks' dimensions up to approximately $20 \%$. However, a purposeful design of the disks is still essential (see SI, Figure S8 for a counterexample).

Impact on Optoelectronic Properties. We further analyzed the optoelectronic properties of a series of solar cells. In order to observe in the most direct way the effect of the disk coating and possible side effects due to processing, we here compare the optimally coated device to an untreated sample with adapted flat ARC. More comprehensive results are shown at a later stage. The left graph in Figure 4 shows the measured current-voltage characteristics of the untreated and the coated cell with an optimal disk density of $3.0 \mu \mathrm{m}^{-2}$ exhibiting the highest increase in $J_{\mathrm{SC}}$ (for details on IV characterization, see Methods). The extracted parameters characterizing these cells are shown in Table 1 . The short circuit current of the coated cell was improved significantly and increased by $14.0 \%_{\text {rel }}$ in comparison to the untreated sample. Due to the increase of current at the maximum power point $I_{\mathrm{MP}}$, the efficiency of the cell $\eta=P_{\max } / P_{\text {in }}$ was increased by $10.4 \%_{\text {rel }}$, with the nominal incident power $P_{\text {in }}=1.521 \mathrm{~W}$. The fill factor $F F=P_{\max } /\left(V_{\mathrm{OC}} \cdot I_{\mathrm{SC}}\right)$ of the coated cell was found to be $2.7 \%_{\text {abs }}$ lower than the one of the untreated sample. However, this is not stemming from the properties of the coating, but rather the higher $V_{\mathrm{MP}}$ of the untreated cell. This can be attributed to the untreated sample not being subjected to any processing, which could potentially cause a small 

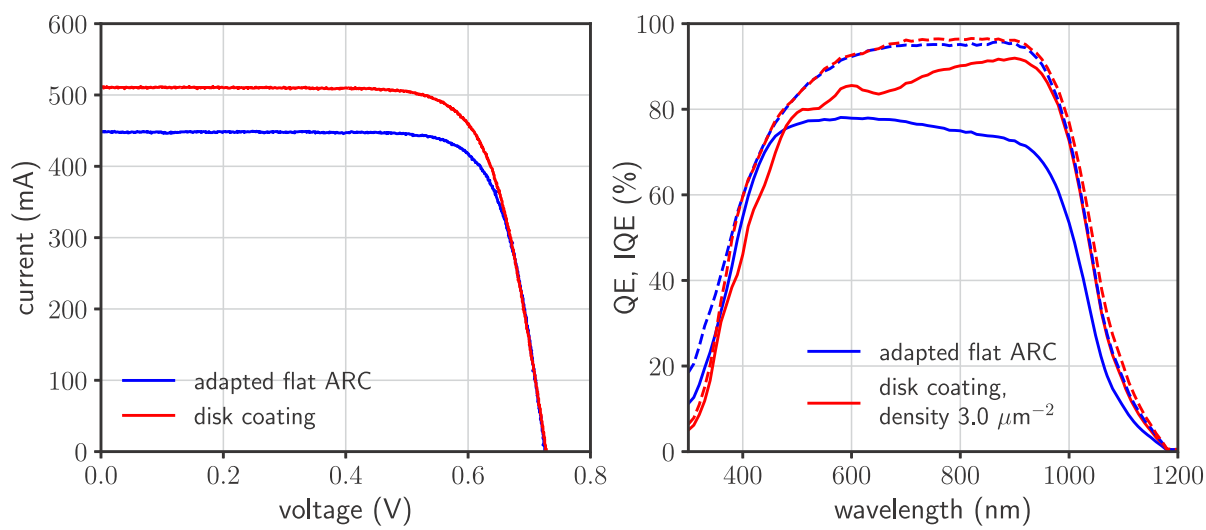

Figure 4. (Left) Current-voltage characteristics of the untreated (adapted flat ARC) sample and coated sample. (Right) External (solid lines) and internal (dashed lines) quantum efficiencies.

Table 1. Cell Parameters Comparing Untreated (Adapted Flat ARC) and the Best Disk Coated Sample, with the Estimated Uncertainty for the Current from Repeated Measurements of Around 0.5\%

\begin{tabular}{lcc} 
& untreated sample & disk coating \\
$I_{\mathrm{SC}, \text { meas }}(\mathrm{mA})$ & 448 & 511 \\
$J_{\mathrm{SC}}\left(\mathrm{mA} / \mathrm{cm}^{2}\right)$ & 29.5 & 33.6 \\
$V_{\mathrm{OC}}(\mathrm{V})$ & 0.726 & 0.728 \\
$I_{\mathrm{MP}}(\mathrm{mA})$ & 420 & 473 \\
$V_{\mathrm{MP}}(\mathrm{V})$ & 0.598 & 0.586 \\
$P_{\max }(\mathrm{W})$ & 0.251 & 0.277 \\
$\eta(\%)$ & 16.5 & 18.2 \\
FF $(\%)$ & 77.2 & 74.5 \\
\hline
\end{tabular}

reduction in $V_{\mathrm{MP}}$ of the coated sample. However, the cell parameters determined from IV characteristics strongly indicate the overall positive effect of the coating, boosting short-circuit current and efficiency of the device.

We characterized the spectral behavior of the coated solar cell with respect to the untreated sample (adapted flat ARC) by measuring external (QE) and internal (IQE) quantum efficiencies (see Methods), shown in the right graph in Figure 4. As expected, the coated cell with optimal disk density shows significant improvement in QE in the most part of the considered spectral region in comparison with the adapted flat ARC sample which has the same ITO thickness of $50 \mathrm{~nm}$. Note that the QE of the latter will not be directly comparable to the QE of a standard flat ARC (see SI, Figure S3) due to the mismatch of reflectance and IQE. The QE of a disk coated device is lower than that of the adapted flat ARC cell only at wavelengths below $500 \mathrm{~nm}$. The region between $\lambda_{0}=350 \mathrm{~nm}$ and $\lambda_{0}=500 \mathrm{~nm}$ is the only part of the spectrum where the reflectance of the coated cell is higher than the reflectance of adapted flat ARC cell (see Figure 3), which leads to higher QE of the latter. Additionally, the $\mathrm{TiO}_{2}$ disks introduce some parasitic absorption in the wavelength range below $\lambda_{0}=380$ $\mathrm{nm}$.

To exclude the effect of the reflection losses in the analysis, we considered the IQE of these cells. Starting from $\lambda_{0}=380$ $\mathrm{nm}$, both devices show comparable performance within measurement uncertainty. Only at shorter wavelengths, the IQE of the coated cell is lower than the IQE of the flat adapted ARC cell due to parasitic absorption in the disks.
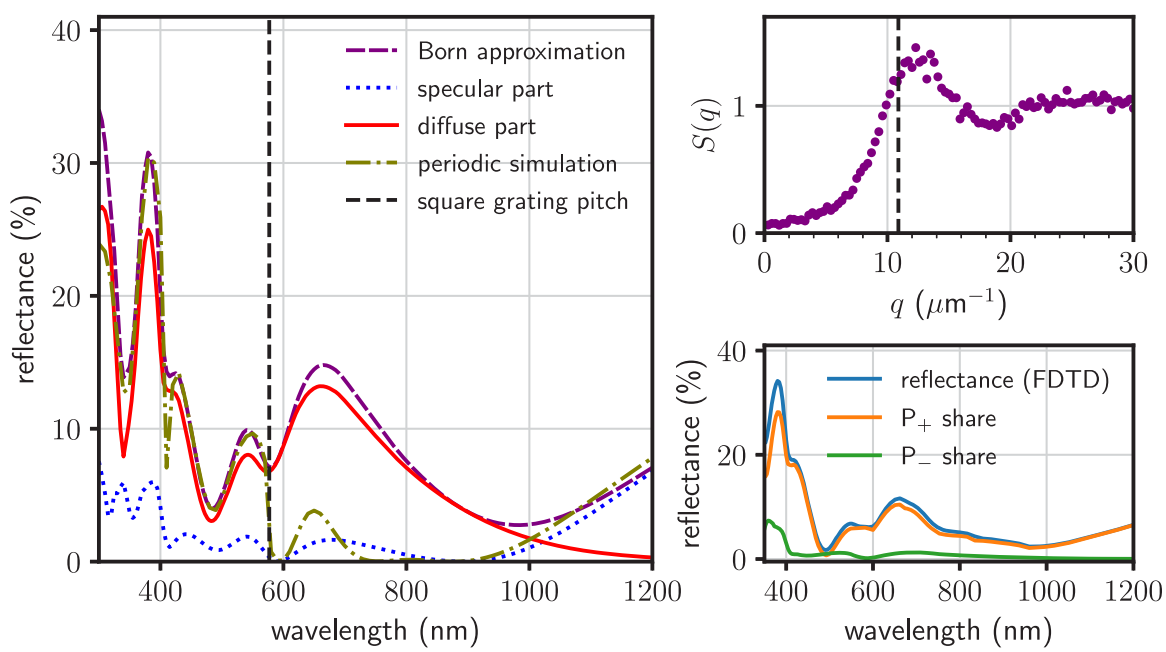

Figure 5. (Left) Decomposition of total reflectance (calculated via Born approximation) into specular and diffuse parts, and comparison to a disk pattern in a square array (with grating pitch of $577 \mathrm{~nm}$ ). (Right, top) Structure factor of the disk pattern calculated from a digitized SEM image. The dashed line marks $q$ corresponding to $577 \mathrm{~nm}$ vacuum wavelength. (Right, bottom) Simulated reflectance via finite-difference time-domain (FDTD) method of a disordered disk arrangement similar to the experiment and decomposition into normalized reflected powers of fields of positive and negative helicity, $\mathrm{P}_{+}$and $\mathrm{P}_{-}$, respectively. The incident field had negative helicity (right circular polarized light). 
Specular and Diffuse Reflectance. In a previous work by Slivina et al., it was numerically shown that periodic disk arrangements can significantly reduce reflectance losses in a solar cell. ${ }^{28}$ Using the same finite element approach, we rigorously simulated the reflectance of a periodic structure with equivalent disk density, based on a disk model with identical parameters, as in the disordered case. In passing we note that, for the Born approximation that describes the disordered sample, we only considered the real part of the refractive index for $\mathrm{TiO}_{2}$. This is not a severe assumption, as the imaginary part of $\mathrm{TiO}_{2}$ anyhow is mostly small in the spectral region of interest. These results compared to results obtained for the disordered sample are shown in Figure 5 (left). Comparison to experimental results are shown in Figure 3. At wavelengths below $577 \mathrm{~nm}$, the Born approximation and periodic simulation are almost congruent and both agree reasonably well with the measured disk sample, except for wavelengths below $350 \mathrm{~nm}$. In this range, absorption in the $\mathrm{TiO}_{2}$ disks becomes significant, which is not included for the Born approximation, hence, the deviation.

The wavelength range above $577 \mathrm{~nm}$, where we observe the strongest deviation between a periodic simulation and the Born approximation, is also of great importance to the device performance due to the strong solar irradiance in this range. To further elucidate the increased reflectance above $577 \mathrm{~nm}$, we decompose the calculated reflectance via Born approximation (see eq 10 in Methods and Figure 1) into specular and diffusive parts, as shown in Figure 5 (left). The diffusive contribution by far outweighs the specular, which is particularly true in the range from 600 to $1000 \mathrm{~nm}$. From eq 12 it is clear that scattering and therefore diffusive reflection can only occur when $q=k_{0} \sin \theta$ is satisfied and $S(q) \neq 0$. The structure factor $S(q)$ for the disordered particle pattern (Figure 5, right, top) peaks at around $q \approx 12.5 \mu \mathrm{m}^{-1}$ and for large $q$ converges to 1 . Toward smaller $q$ it decays from the peak value down to about $S_{\min } \approx 0.06$ for reasons discussed elsewhere in more detail. ${ }^{18,35,41}$ In Figure 5 (right, top) we also marked the length of the wave vector corresponding to a vacuum wavelength $\lambda_{\mathrm{c}}=577 \mathrm{~nm}$, which can only be affected (scattered) by structure factor components with $q \leq k\left(\lambda_{\mathrm{c}}\right)$. At $q=k\left(\lambda_{\mathrm{c}}\right)$, the structure factor $S(q)$ is relatively large; however, for longer wavelengths, the relevant range for scattering is restricted to even smaller $q$. We, therefore, observe reduced scattering for longer wavelengths due to $S(q)$ being small at small $q$. Note, that for a periodic grating with the smallest grating vector $|\mathbf{q}|=q_{\mathrm{c}}=2 \pi / 577 \mathrm{~nm}$, all $S\left(q<q_{\mathrm{c}}\right)$ vanish. In contrast to our disordered sample, the periodic disk arrangement from the simulation (Figure 5, left) does not support any propagating modes besides the zeroth order, hence, the lower reflectance starting from $577 \mathrm{~nm}$.

The possible exact rotational symmetries as initially required for helicity preservation in an ordered system (see the first section of this paper) are lost in a disordered system, hereby not meeting one of the conditions for zero reflection. Additionally, nonspecular reflections are possible due to the diffuse components allowed by $S(q) \neq 0$, but the theorem for zero reflection $^{29}$ is only applicable to the specular component. Crucially though, disorder if tailored appropriately can also end up overcoming both obstacles. A subclass of point configurations where

$$
S(0<q \leq Q)=0
$$

in the range $Q$ around the origin is called stealthy hyperuniform. ${ }^{26}$ Periodic systems trivially meet this condition, however, also disordered systems can be tailored to do so and are then called disordered stealthy hyperuniform. They do not possess Bragg peaks and are fully isotropic and therefore exhibit effective continuous rotational symmetry. ${ }^{42}$ Then, on the one hand, the diffuse component of the total reflection is completely suppressed and on the other hand, the continuous rotational symmetry $2 \pi / n$ with $n \rightarrow \infty$ meets the requirement for zero specular reflection. Note that condition (1) is in many cases a weaker requirement than periodicity.

To discuss the results in Figure 5 (left) in light of this knowledge, we also calculated the optical response of the disordered structure upon plane-wave illumination at normal incidence with right-hand circular polarization, that is, negative helicity. These calculations were performed using the finitedifference time-domain (FDTD) method (see Methods). The backscattered light (reflectance) was decomposed into positive and negative helicity components, $\mathrm{P}_{+}$and $\mathrm{P}_{-}$(Figure 5, right, bottom), similar to the decomposition for periodic structures in ref 28. At wavelengths shorter than $577 \mathrm{~nm}$, which corresponds to the square lattice constant, the periodic system scatters in multiple diffraction orders, which greatly contribute to the mixed helicity of the reflected light (see ref 28). For the disordered system, a continuum of scattering channels is open with the same effect, that is, $\mathrm{P}_{+}$and $\mathrm{P}_{-}$contribute to the reflectance (Figure 5, right, bottom). However, the differences in reflectance between periodic and disordered systems are more pronounced in the wavelength region between $\lambda_{0}=577$ $\mathrm{nm}$ and $\lambda_{0}=1000 \mathrm{~nm}$. For the periodic system, only the zeroth diffraction order is present, and all reflected light is of flipped helicity $\left(P_{+}\right.$, see ref 28$)$. It follows that the backscattered power will decrease as the degree of helicity preservation of the system increases. In contrast, $S(q)$ of the disordered system is still considerably higher than 0 (e.g., $S(q=2 \pi /(800 \mathrm{~nm})) \approx$ $0.48)$, and the main contribution to reflectance is again the diffuse part, thereby allowing reflected light of the same helicity as the incident light. Nevertheless, the bottom right panel of Figure 5 shows that the reflectance is mostly dominated by the component of flipped helicity. The differences between the disordered system and the periodic system diminish as the relative contribution of the specular part to the backscattered light increases (in particular, from $\lambda_{0}=1000 \mathrm{~nm}$ on). For such longer wavelengths, $S(q)$ is decaying (e.g., $S(q=2 \pi /(1200$ $\mathrm{nm})) \approx 0.18$ ), and we enter the regime where the disordered system is sufficiently stealthy. The reflection losses at $\lambda_{0}=$ $1200 \mathrm{~nm}$ are similar to the losses at $800 \mathrm{~nm}$. But in the $\lambda_{0}=$ $1200 \mathrm{~nm}$ region, the reflection losses are almost exclusively due to the specular part of the reflectance with flipped helicity $\left(\mathrm{P}_{+}\right)$, and the reflected power corresponding to the scattered field of the same helicity as the incident field $\left(\mathrm{P}_{-}\right)$approaches zero, resembling the helicity decomposition of the periodic system. ${ }^{28}$ It is in this long wavelength region that the reflection loss for our disordered system has a one-to-one relation to the changed helicity component. We suspect that reducing $S(q)$ also for larger $q$ would extend such a one-to-one relation toward lower wavelength regions. Additionally, the results indicate that, even in the diffusive region of the spectrum, improved helicity preservation of the system would result in reduced reflectance, although not in a one-to-one relation. Note that the effect of the decaying $S(q)$ is complemented by a decreasing scattering cross-section of the individual disk with increasing wavelength. 
The delicate interplay between $S(q)$ and individual scattering response is analyzed in depth in ref 18 .

Given the other device characteristics remain largely intact after disk fabrication, the short circuit current is the single most relevant parameter to assess the performance of the coating. Assuming the internal quantum efficiency of the disk coated sample also remains unchanged compared to an untreated cell with $\operatorname{IQE}_{\text {ref }}\left(\lambda_{0}\right)$ (see Figure 4 ), we can estimate the short circuit current density of a solar cell

$$
J_{\mathrm{SC}}=e \int_{300 \mathrm{~nm}}^{1200 \mathrm{~nm}} \phi_{\mathrm{AM} 1.5 \mathrm{G}}\left(\lambda_{0}\right) \mathrm{IQE}_{\mathrm{ref}}\left(\lambda_{0}\right)\left(1-R\left(\lambda_{0}\right)\right) \mathrm{d} \lambda_{0}
$$

where $\phi_{\mathrm{AM} 1.5 \mathrm{G}}\left(\lambda_{0}\right)$ denotes the incident photon flux and $e$ is the elementary charge. Table 2 shows calculated $J_{S C}$ values from reflectance curves of the Born approximation and the periodic simulation.

Table 2. Calculated Current Densities and Current Losses for the Disordered Sample (from Reflectance Calculation via Born Approximation) and Periodic Sample (Rigorous Simulation of Reflectance)

\begin{tabular}{|c|c|c|c|c|}
\hline & \multicolumn{2}{|c|}{ current density $\left(\mathrm{mA} / \mathrm{cm}^{2}\right)$} & \multicolumn{2}{|c|}{$\begin{array}{c}\text { current loss } \\
\left(\mathrm{mA} / \mathrm{cm}^{2}\right) \\
\lambda_{0}>577 \mathrm{~nm}\end{array}$} \\
\hline & total & $\lambda_{0}>577 \mathrm{~nm}$ & specular & diffuse \\
\hline Born approximation & 33.44 & 25.15 & -0.23 & -2.01 \\
\hline periodic simulation & 35.42 & 27.26 & -0.30 & 0 \\
\hline$\Delta J_{\mathrm{SC}}$ & -1.98 & -2.11 & 0.07 & -2.01 \\
\hline
\end{tabular}

As expected, the current density is lower for the disordered sample (Born approximation) due to the higher reflectance. Considering only the current contribution from wavelengths $\lambda_{0}$ $>577 \mathrm{~nm}$, we can infer that the difference in total current is accounted for by differences in reflectance in this range. However, the current loss due to specular reflectance beyond $577 \mathrm{~nm}$ is comparably low for both cases, while the loss due to diffusive reflectance at the disordered sample is about -2.01 $\mathrm{mA} / \mathrm{cm}^{2}$. Therefore, the difference in performance of both antireflective structures is primarily a consequence of the nonzero structure factor components in the low $q$ range.

The simulated periodic system has been optimized with the prerequisite of a square grating, albeit the optimal parameters may not be the same for the disordered arrays of disks. As shown above, the share of absorbed incident photons $(1-R)$ is directly related to the short circuit current density $J_{S C}$ which is therefore the single most relevant device parameter in judging the antireflective coating. For the reflectance $R$ we use the calculated curves such as shown in Figure 5. Instead of using structure factors $S(q)$ from fabricated samples, however, we predicted a series of particle patterns within the bounds of the deposition technique (see ref 35) and forecast the response of those patterns.

Figure 6 shows the calculated $J_{S C}$ of a series of such predicted patterns with varying disk density. The highest current is achieved at a disk density of about $3.0 \mu \mathrm{m}^{-2}$. This is the density previously discussed in depth. A series of experiments with varying disk density is also shown in Figure 6 along with the measured current density for a standard flat ARC. The measured data essentially confirms the trends as well as the optimum of the prediction. The highest measured current densities are slightly lower than calculated, however,

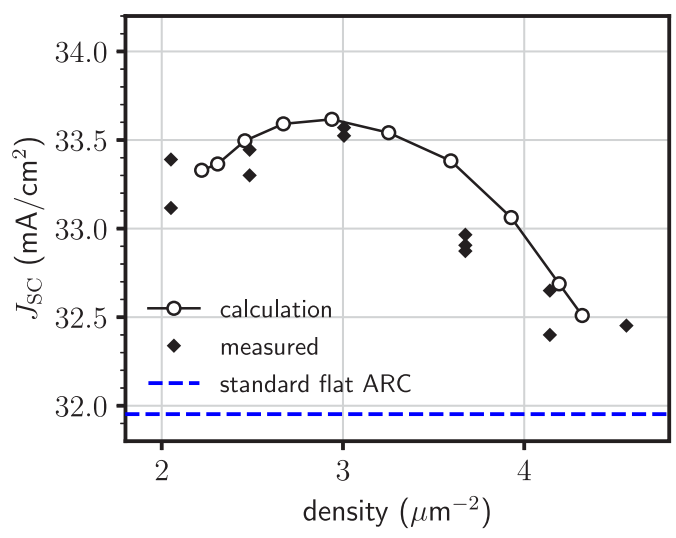

Figure 6. Short circuit current density versus disk density for simulated correlated disorder disk patterns (open circles, solid line is a guide to the eyes) and measured short circuit current densities (black squares). Additionally, the median current density of a series of samples with standard flat antireflective coating ( $80 \mathrm{~nm} \mathrm{ITO)} \mathrm{is}$ shown (blue dashed line).

given the differences between measured and calculated reflectance (Figure 3), some deviations were to be expected. Also, there is some degree of uncertainty in predicting the particle patterns from which reflectances are calculated. ${ }^{35}$ Namely, the deposition model does not include any aggregation of particles which tends to increase the values of $S(q)$ for small $q$, as discussed in ref 18 in more detail. Therefore, we expect higher diffuse scattering contributions at wavelengths $\lambda_{0}>600 \mathrm{~nm}$ and consequently higher current losses in the measurement compared to the predicted pattern, as discussed above.

Notably, for all considered disk densities the current is higher for both calculation and measurement compared to the standard flat ARC reference, which corresponds to the measured reflectance in Figure 3. The highest measured difference of about $1.6 \mathrm{~mA} / \mathrm{cm}^{2}\left(+5.1 \%_{\text {rel }}\right)$ is found at $3.0 \mu \mathrm{m}^{-2}$. Considering the change in current for the samples before and after disk deposition, the increase is even more decisive with $4.1 \mathrm{~mA} / \mathrm{cm}^{2}\left(+14.0 \%_{\text {rel }}\right)$. Another benchmark would be a double ARC stack, consisting of a $\mathrm{MgF}_{2}$ layer on top of the ITO layer (each with optimized thickness), for which we estimate a current density increase of $1.5 \mathrm{~mA} / \mathrm{cm}^{2}$ $\left(+4.7 \%_{\text {rel }}\right)$ compared to the standard flat $\mathrm{ARC}^{43,44}$ (see SI, Figure S7 for reflectance and details). However, the effect of encapsulation of the solar cell in a module tends to annihilate the advantage of the double ARC, while this is not the case for an optimized disk structure. ${ }^{34}$ Additionally, the total current density for the periodic simulation in Table 2 and the corresponding discussion suggest that even higher current is possible to reach with the disk structure by further reducing the structure factor for small $q$.

\section{CONCLUSION}

We have demonstrated the successful implementation of a $\mathrm{TiO}_{2}$ dielectric disk-based disordered AR coating in solar cells of standard industrial design by using a scalable bottom-up technique. ${ }^{18}$ Robustness of this deposition technique with respect to the surface morphology allows to avoid special preparation of the substrate, which, in addition to scalability, makes it an attractive technique to fabricate disordered particle coatings. Our measurements show significant reflection suppression in the spectral range relevant for solar cells, and 
we observe $5.1 \%_{\text {rel }}$ improvement of the short-circuit current in comparison to a standard (optimized for AM1.5G irradiance) flat ARC reference device.

Additionally, we corroborate our experimental results with a theoretical model based on Born's first approximation which takes into account the disordered nature of the disk coating. Our results show that most of the reflected light is of changed helicity with respect to the helicity of the incident light. As the stealthiness of the disk coating increases $(S(q)$ decreases) with increasing wavelength, the reflectance becomes equal to the power of the changed helicity component. These findings support the purposeful design of the coatings based on dielectric high-index nanoparticles targeting helicity preservation, even though the existence of nonspecular components reduces the effectiveness as compared to a periodic system in the nondiffracting region. ${ }^{28}$ Moreover, our design is exemplary and is neither restricted to the materials used nor to a particular solar cell stack and can be applied to other systems.

\section{METHODS}

Sample Fabrication. The experiment is based on industrial type heterojuntion techology (HJT) solar cells fabricated from $150 \mu \mathrm{m}$ Czochralski silicon (cSi) wafers. Instead of a pyramidal standard texture, a mostly flat surface was achieved by adapting the wet-chemical damage etch process. The HJT solar cells used in the experiment were rear emitter cells that were passivated with amorphous silicon $(\mathrm{aSi})$. The thicknesses of the front passivation (intrinsic aSi and $\mathrm{n}^{+}$ doped $\mathrm{aSi}$ ) and ITO layers can be found in ref 28 and similar passivation (intrinsic aSi and $\mathrm{p}^{+}$doped) and ITO layers were applied to the rear side. Two alternative types of front ITO layers were used. The reference devices with $80 \mathrm{~nm}$ ITO layers were optimized for efficiency under standard conditions. The disk coating was based on $50 \mathrm{~nm}$ ITO layers with higher doping levels and carrier concentration to compensate for the loss in conductivity due to the lower thickness. A total of 81 sample with dimensions $39 \times 39 \mathrm{~mm}^{2}$ were laser-cut from fully processed $156 \mathrm{~mm}$ solar cells (including contact fingers) to match the processing capabilities for the subsequent disk fabrication process.

Details of the disk fabrication have been published elsewhere. ${ }^{18}$ Briefly, it is based on self-organizing colloidal particles that are deposited onto a functionalized surface and the patterns thereof are subsequently transferred to a $\mathrm{TiO}_{2}$ layer via RIE. On top of the front ITO layer, $\mathrm{TiO}_{2}$ layers of $105.5 \mathrm{~nm}$ thickness were deposited via ALD at $120{ }^{\circ} \mathrm{C}$ using alternating pulses of titanium tetrachloride $\left(\mathrm{TiCl}_{4}\right)$ and water (1800 pulses each) in a Beneq TFS-200 thermal ALD tool. Additionally, a thinner $16.8 \mathrm{~nm}$ layer of aluminum oxide $\left(\mathrm{Al}_{2} \mathrm{O}_{3}\right)$ was deposited $\left(120{ }^{\circ} \mathrm{C}, 150\right.$ pulses of trimethylaluminum (TMAl) and water, each) as functionalization for the particle deposition process. The samples were exposed to a dispersion of $350 \mathrm{~nm}$ diameter poly(methyl methacrylate) (PMMA) particles (microParticles Berlin GmbH, Germany), which formed an evenly distributed arrangement on the surface. $^{35}$ The particles were tempered briefly $(30 \mathrm{~min}$ at $135^{\circ} \mathrm{C}$ ) for improved covering of the surface, such that they could serve as an etch mask for RIE. This process step was likely the reason for the slight increase of disk diameter (370 $\mathrm{nm}$ ) compared to particle diameter. The etch process was carried out in two steps: First, the $\mathrm{Al}_{2} \mathrm{O}_{3}$ layer was structured using a Chlorine-containing plasma. Second, the structured $\mathrm{Al}_{2} \mathrm{O}_{3}$ was used as hard-mask in a fluorine-based process to selectively etch $\mathrm{TiO}_{2}$. The ITO contact beneath is only weakly etched in Fluorine-plasmas and served as an etch-stop layer.

IV Characterization. For each of the laser-separated samples (see Sample Fabrication) the IV characterization was performed under standard test conditions of $1 \mathrm{~kW} / \mathrm{m}^{2}$ mean irradiance and a cell temperature in the range of $25 \pm 1$ ${ }^{\circ} \mathrm{C}$ (adjusted via calibration) using a dedicated setup for bus barless cells ${ }^{45}$ in order to compare them (see Table 1) with the focus on $I_{\mathrm{SC}}$, which is influenced by the coating. The coated cells were characterized within one month after separation from full-sized cells, however, the smaller than usual samples led to unstable voltage readings due to a mechanical problem with the voltage probe wire, while not affecting the current measurement. For those samples discussed in terms of voltage, we repeated the IV characterization within four months after separation. The current was scaled in order to account for handling of the samples and differences in the calibration, as well as the possible degradation of the samples in the time span between the measurements. The scaling factor $a$ is the ratio of short circuit currents $I_{\mathrm{SC}, i}$ :

$$
a=\frac{I_{\mathrm{SC}, 1 \mathrm{~m}}}{I_{\mathrm{SC}, 4 \mathrm{~m}}}
$$

where indices $i=1 \mathrm{~m}$ and $i=4 \mathrm{~m}$ denote the delays between separation and measurement. The corrected currents presented in Figure 4 and in Table 1 were then obtained by dividing the measured values with $a$, which corresponds to an average change (reduction) in the short-circuit current $\Delta_{\text {rel }} I_{S C}=1-a$ $=0.64 \%$ within three months between the measurements, which is slightly above the estimated uncertainty for the current.

Reflectance and Quantum Efficiencies. Reflectance was measured using a PerkinElmer Lambda $750 \mathrm{UV} /$ vis/NIR spectrophotometer. QE measurements were performed using PV Measurements, Inc. Solar Cell Spectral Response/QE/ IPCE Measurement System, Model QEX7 with black back sheet as a background. In order to account for signal instabilities during the QE measurement, we scaled the raw data with respect to $J_{\mathrm{SC}}$, obtained from the IV characteristic. This was carried out by calculating the short circuit current from the $\mathrm{QE}$ data, $J_{\mathrm{SC}, \mathrm{QE}}$, and subsequently calculating the scaling factor $b=J_{\mathrm{SC}, \mathrm{QE}} / J_{\mathrm{SC}}$, where $J_{\mathrm{SC}}$ is the value taken from Table 1:

$$
J_{\mathrm{SC}, \mathrm{QE}}=e \int_{300 \mathrm{~nm}}^{1200 \mathrm{~nm}} \phi_{\mathrm{AM} 1.5 \mathrm{G}}\left(\lambda_{0}\right) \cdot \mathrm{QE}\left(\lambda_{0}\right) \mathrm{d} \lambda_{0}
$$

where $e$ is the elementary charge and $\phi_{\mathrm{AM} 1.5 \mathrm{G}}$ is the incident photon flux. The scaled external $\left(\mathrm{QE}_{\mathrm{s}}\right)$ and internal $\left(\mathrm{IQE}_{\mathrm{s}}\right)$ quantum efficiencies were then calculated using the following equations:

$$
\begin{aligned}
& \mathrm{QE}_{\mathrm{s}}=\mathrm{QE} / b \\
& \mathrm{IQE}_{\mathrm{s}}=\frac{\mathrm{QE}_{\mathrm{s}}}{1-R}
\end{aligned}
$$

where $R$ is the measured reflectance.

Numerical Methods and Material Information. The numerical simulations discussed in this paper were done using the finite element method (FEM) with commercial software JCMsuite. $^{46}$ Refractive indices for $\mathrm{cSi}$ and ITO used in calculations were taken from literature. ${ }^{47,48}$ Refractive index data for aSi, $\mathrm{TiO}_{2}$, and $\mathrm{Al}_{2} \mathrm{O}_{3}$ were obtained by means of 
ellipsometry, and corresponding $n$ and $k$ values are provided in the SI. ${ }^{49}$

Additional simulations of a $3 \times 3 \mu \mathrm{m}^{2}$ unit cell containing 27 disks under periodic boundary conditions were performed using the FDTD method (free software Meep ${ }^{50}$ ). The disks were arranged to match the experimentally determined structure factor $^{51}$ (see SI for details).

Calculation of Reflectance. The disks are arranged in a two-dimensional pattern in a plane perpendicular to the incident field propagating in $z$-direction. Coupling between disks is therefore considerably lower than in three-dimensional pattern of similar scatterer density, and we consider the individual disks only to be excited by the incident field $\boldsymbol{E}_{\text {in }}=\boldsymbol{E}_{0} e^{i\left(k_{0} z-\omega t\right)}$ and employ the first Born approximation ${ }^{52}$ to calculate the scattering response of our disk layer. ${ }^{18}$ In the far field, the scattered electric field of an individual scatterer has the form of a spherical wave $\mathrm{e}^{53}$

$$
\boldsymbol{E}_{\mathrm{s}}(\boldsymbol{r})=E_{0} \frac{e^{i k \boldsymbol{r}}}{r} \boldsymbol{f}
$$

where the time dependence $e^{-i \omega t}$ has been omitted. The form factor $\boldsymbol{f}=\boldsymbol{f}\left(\hat{\boldsymbol{E}}_{0}, \boldsymbol{k}_{0}, \boldsymbol{k}\right)$ describes the normalized field amplitude for an incident field with polarization $\hat{\boldsymbol{E}}_{0}=\boldsymbol{E}_{0} /\left|\boldsymbol{E}_{0}\right|$, where $\boldsymbol{k}_{0}$ and $\boldsymbol{k}$ denote the incoming and scattered wave vectors, respectively. In this work, we obtain $f$ via finite element simulation, using a model with identical parameters to those of the periodic simulation, except for neglecting absorption by the disks for numerical reasons (see below).

Considering now only the scattering components perpendicular to the disk plane in forward direction with $k^{+}=n_{\mathrm{Si}} k_{0} \boldsymbol{e}_{z}$ (silicon half-space) and backward direction $\boldsymbol{k}^{-}=-k_{0} \boldsymbol{e}_{z}$ (air half-space) of an individual disk illuminated with a linearly polarized plane wave with $k_{0} \boldsymbol{e}_{z}$, we can use the angular spectrum (eq 7) in its asymptotic limit to calculate specular transmission and reflection ${ }^{54}$

$$
\begin{aligned}
& T_{\text {spec }}=n_{\mathrm{Si}}\left|t \hat{\boldsymbol{E}}_{0}+\rho \frac{2 \pi}{i\left|\boldsymbol{k}^{+}\right|} \boldsymbol{f}\left(\boldsymbol{k}=\boldsymbol{k}^{+}\right)\right|^{2} \\
& R_{\text {spec }}=\left|r \hat{\boldsymbol{E}}_{0}+\rho \frac{2 \pi}{i\left|\boldsymbol{k}^{-}\right|} \boldsymbol{f}\left(\boldsymbol{k}=\boldsymbol{k}^{-}\right)\right|^{2}
\end{aligned}
$$

where $t$ and $r$ denote the Fresnel transmission and reflection coefficients of the planar layer stack and $\rho$ is the density of the disks. Since the interaction between the disks is neglected, $T$ and $R$ do not depend on the particular arrangement of disks, but only on the density $\rho$. All scattered fields of the individual disks in normal direction are in phase and interfere with $t \hat{\mathbf{E}}_{0}$ and $r \hat{\boldsymbol{E}}_{0}$ of the planar stack if polarizations match. If absorption within the scattering structure is neglected, energy conservation demands that

$$
1-T_{\text {spec }}-R_{\text {spec }}=T_{\text {diff }}+R_{\text {diff }}=P_{\text {diff }}
$$

with $T_{\text {diff }}$ and $R_{\text {diff }}$ denoting diffusive contributions to transmission and reflection, and $P_{\text {diff }}$ is the normalized scattered power excluding specular components. On the other hand, the scattered power must be equivalent to the integral of the angular resolved scattering (ARS) over the unit sphere $\Omega$ (excluding $\mathrm{d} \Omega=\mathrm{d} \Omega\left(\boldsymbol{k}=\boldsymbol{k}^{ \pm}\right)$), with $P_{\text {diff }}=\int_{\Omega} \mathrm{ARS}$ $\mathrm{d} \Omega$. The ARS now is obtained by modulating the individual scattering response $|f|^{2}$ with the structure factor $S\left(q=k_{\|}\right)$, where $k_{\|}$is the projection of the scattered wave vector $k$ to the disk pattern plane and needs to be scaled in order to satisfy eq 10:

$$
\mathrm{ARS}=\frac{P_{\mathrm{diff}}}{\int_{\Omega}|\boldsymbol{f}|^{2} S \mathrm{~d} \Omega} \cdot|\boldsymbol{f}(\boldsymbol{k})|^{2} S\left(k_{\|}\right) \quad \boldsymbol{k} \neq \boldsymbol{k}^{ \pm}
$$

The diffusive part of the reflectance is obtained by integrating over the half-sphere $\Omega^{\prime}$ in air,

$$
R_{\mathrm{diff}}=\int_{\Omega^{\prime}} \mathrm{ARS} \mathrm{d} \Omega \quad \boldsymbol{k} \neq \boldsymbol{k}^{-}
$$

For the periodic case, reflectance was calculated from the ratio of the power scattered into the upper half-space to the power of the incident field as

$$
R_{\text {periodic }}=\frac{\sum_{\mathbf{k}_{\mathrm{r}}}\left|\tilde{\boldsymbol{E}}\left(k_{\mathrm{r}, x}, k_{\mathrm{r}, y}\right)\right|^{2} \cdot \cos \left(\theta_{\mathrm{r}}\right)}{\left|\boldsymbol{E}_{0}\right|^{2}}
$$

where $k_{r}$ are the wave vectors of the reflected electromagnetic waves, $\theta_{\mathrm{r}}$ are the angles between these wave vectors and the $z$ axis, $E_{0}$ is the amplitude of a normally incident and linearly polarized plane wave, and the angular spectrum of the scattered field $\tilde{\boldsymbol{E}}\left(k_{\mathrm{r}, x}, k_{\mathrm{r}, y}\right)$ is determined via Fourier transform of the field in real space obtained from full-wave simulations.

\section{ASSOCIATED CONTENT}

\section{(s) Supporting Information}

The Supporting Information is available free of charge at https://pubs.acs.org/doi/10.1021/acsphotonics.1c00601.

Disks deposited on a textured surface as demonstration of robustness of the fabrication technique; Optical material data used for simulations; Quantum efficiencies for various disk densities and a reference device; Short circuit current density before/after comparison of diskcoated cells; Comparison of double ARC with standard flat ARC; Reciprocal space optimized sample for FDTD simulations; Results of the FDTD simulation for disks with increased height (PDF)

\section{AUTHOR INFORMATION}

\section{Corresponding Authors}

Peter M. Piechulla - Institute of Physics, Martin Luther University Halle-Wittenberg, 06120 Halle, Germany; (1) orcid.org/0000-0002-9495-1709;

Email: peter.piechulla@physik.uni-halle.de

Evgeniia Slivina - Institute of Theoretical Solid State Physics, Karlsruhe Institute of Technology, 76131 Karlsruhe, Germany; (1) orcid.org/0000-0003-4645-1491; Email: evgeniia.slivina@kit.edu

\section{Authors}

Derk Bätzner - Meyer Burger Research AG, Hauterive 2068, Switzerland

Ivan Fernandez-Corbaton - Institute of Nanotechnology, Karlsruhe Institute of Technology, 76344 EggensteinLeopoldshafen, Germany; $\odot$ orcid.org/0000-0003-28345572

Prerak Dhawan - Institute of Theoretical Solid State Physics, Karlsruhe Institute of Technology, 76131 Karlsruhe, Germany 
Ralf B. Wehrspohn - Institute of Physics, Martin Luther University Halle-Wittenberg, 06120 Halle, Germany

Alexander N. Sprafke - Institute of Physics, Martin Luther

University Halle-Wittenberg, 06120 Halle, Germany

Carsten Rockstuhl - Institute of Theoretical Solid State Physics, Karlsruhe Institute of Technology, 76131 Karlsruhe, Germany; Institute of Nanotechnology, Karlsruhe Institute of Technology, 76344 Eggenstein-Leopoldshafen, Germany

Complete contact information is available at: https://pubs.acs.org/10.1021/acsphotonics.1c00601

\section{Author Contributions}

\#These authors contributed equally to this work.

\section{Notes}

The authors declare no competing financial interest.

\section{ACKNOWLEDGMENTS}

This work was funded by the Deutsche Forschungsgemeinschaft (DFG) through Program DFG-SPP 1839 "Tailored Disorder", second period (RO 3640/6-2 and WE4051/19-2, Project 278744673), under the Germany's Excellence Strategy via the Excellence Cluster 3D Matter Made to Order (No. EXC-2082/1-390761711), and the KIT through the "Virtual Materials Design" (VIRTMAT) Project. The authors are grateful to the company JCMwave for their free provision of the FEM Maxwell solver JCMsuite.

\section{REFERENCES}

(1) Shockley, W.; Queisser, H. J. Detailed balance limit of efficiency of $\mathrm{p}-\mathrm{n}$ junction solar cells. J. Appl. Phys. 1961, 32, 510.

(2) Ehrler, B.; Hutter, E. M.; Berry, J. J. The Complicated Morality of Named Inventions. ACS Energy Lett. 2021, 6, 565-567.

(3) Rong, Y.; Hu, Y.; Mei, A.; Tan, H.; Saidaminov, M. I.; Seok, S. I.; McGehee, M. D.; Sargent, E. H.; Han, H. Challenges for Commercializing Perovskite Solar Cells. Science 2018, 361, No. eaat8235.

(4) Galagan, Y. Perovskite Solar Cells: Toward Industrial-Scale Methods. J. Phys. Chem. Lett. 2018, 9, 4326-4335.

(5) Chen, D.; Manley, P. Nanophotonic Light Management for Perovskite-Silicon Tandem Solar Cells. J. Photonics Energy 2018, 8, 022601.

(6) Hou, Y.; et al. Efficient Tandem Solar Cells with SolutionProcessed Perovskite on Textured Crystalline Silicon. Science 2020, 367, 1135-1140.

(7) Schulze, P. S. C.; Bett, A. J.; Bivour, M.; Caprioglio, P.; Gerspacher, F. M.; Kabakl1, Ö. Ş.; Richter, A.; Stolterfoht, M.; Zhang, Q.; Neher, D.; Hermle, M.; Hillebrecht, H.; Glunz, S. W.; Goldschmidt, J. C. 25.1\% High-Efficiency Monolithic Perovskite Silicon Tandem Solar Cell with a High Bandgap Perovskite Absorber. Sol. RRL 2020, 4, 2000152.

(8) Stavroulakis, P. I.; Boden, S. A.; Johnson, T.; Bagnall, D. M. Suppression of Backscattered Diffraction from Sub-Wavelength 'Moth-Eye' Arrays. Opt. Express 2013, 21, 1-11.

(9) Florescu, M.; Gkantzounis, G. Hyperuniform Disordered Structures for Enhanced Light Absorption. Proc. SPIE 10527 2018, $105270 \mathrm{~J}$.

(10) Sprafke, A.; Pollard, M.; Piechulla, P.; Bagnall, D.; Hoex, B.; Conibeer, G.; Wehrspohn, R. B. Mie resonators as rearside light trapping structures in planar crystalline silicon solar cells. Light, Energy and the Environment 2018 (E2, FTS, HISE, SOLAR, SSL); OSA, 2018; paper OT3C.5, DOI: 10.1364/OSE.2018.OT3C.5.

(11) Yalamanchili, S.; Verlage, E.; Cheng, W.-H.; Fountaine, K. T.; Jahelka, P. R.; Kempler, P. A.; Saive, R.; Lewis, N. S.; Atwater, H. A. High Broadband Light Transmission for Solar Fuels Production Using
Dielectric Optical Waveguides in $\mathrm{TiO} 2$ Nanocone Arrays. Nano Lett. 2020, 20, 502-508.

(12) Garnett, E. C.; Ehrler, B.; Polman, A.; Alarcon-Llado, E. Photonics for Photovoltaics: Advances and Opportunities. ACS Photonics 2021, 8, 61-70.

(13) Donie, Y. J.; Smeets, M.; Egel, A.; Lentz, F.; Preinfalk, J. B.; Mertens, A.; Smirnov, V.; Lemmer, U.; Bittkau, K.; Gomard, G. Light Trapping in Thin Film Silicon Solar Cells via Phase Separated Disordered Nanopillars. Nanoscale 2018, 10, 6651-6659.

(14) Spinelli, P.; Polman, A. Light Trapping in Thin Crystalline Si Solar Cells Using Surface Mie Scatterers. IEEE J. Photovoltaics 2014, 4, 554-559.

(15) Staude, I.; Miroshnichenko, A. E.; Decker, M.; Fofang, N. T.; Liu, S.; Gonzales, E.; Dominguez, J.; Luk, T. S.; Neshev, D. N.; Brener, I.; Kivshar, Y. Tailoring Directional Scattering through Magnetic and Electric Resonances in Subwavelength Silicon Nanodisks. ACS Nano 2013, 7, 7824-7832.

(16) van de Groep, J.; Polman, A. Designing Dielectric Resonators on Substrates: Combining Magnetic and Electric Resonances. Opt. Express 2013, 21, 26285-26302.

(17) Rahimzadegan, A.; Arslan, D.; Suryadharma, R. N. S.; Fasold, S.; Falkner, M.; Pertsch, T.; Staude, I.; Rockstuhl, C. DisorderInduced Phase Transitions in the Transmission of Dielectric Metasurfaces. Phys. Rev. Lett. 2019, 122, 015702.

(18) Piechulla, P. M.; Fuhrmann, B.; Slivina, E.; Rockstuhl, C.; Wehrspohn, R. B.; Sprafke, A. N. Tailored Light Scattering through Hyperuniform Disorder in Self-Organized Arrays of High-Index Nanodisks. Adv. Opt. Mater. 2021, 9, 2100186.

(19) Spinelli, P.; Verschuuren, M. A.; Polman, A. Broadband Omnidirectional Antireflection Coating Based on Subwavelength Surface Mie Resonators. Nat. Commun. 2012, 3, 692.

(20) Spinelli, P.; Lenzmann, F.; Weeber, A.; Polman, A. Effect of EVA Encapsulation on Antireflection Properties of Mie Nanoscatterers for C-Si Solar Cells. IEEE J. Photovoltaics 2015, 5, 559-564. (21) Stevens, L.; Tucher, N.; Höhn, O.; Hauser, H.; Müller, C.; Bläsi, B. Broadband Antireflection Mie Scatterers Revisited-a Solar Cell and Module Analysis. Opt. Express 2019, 27, A524-A535.

(22) Oskooi, A.; Favuzzi, P. A.; Tanaka, Y.; Shigeta, H.; Kawakami, Y.; Noda, S. Partially disordered photonic-crystal thin films for enhanced and robust photovoltaics. Appl. Phys. Lett. 2012, 100, 181110 .

(23) van Lare, M.-C.; Polman, A. Optimized Scattering Power Spectral Density of Photovoltaic Light-Trapping Patterns. ACS Photonics 2015, 2, 822-831.

(24) Pratesi, F.; Burresi, M.; Riboli, F.; Vynck, K.; Wiersma, D. S. Disordered Photonic Structures for Light Harvesting in Solar Cells. Opt. Express 2013, 21, A460-A468.

(25) Tavakoli, N.; Spalding, R. J.; Gkantzounis, G.; Wan, C.; Röhrich, R.; Kontoleta, E.; Florescu, M.; Sapienza, R.; Koenderink, F.; Lladó, E. A. Hyperuniform Designs for Enhanced Light Trapping in Ultrathin Single and Tandem Solar Cells (Conference Presentation). Photonics for Solar Energy Systems VIII; SPIE, 2020; p 113660A, DOI: $10.1117 / 12.2554441$.

(26) Torquato, S. Hyperuniform States of Matter. Phys. Rep. 2018, $745,1-95$.

(27) Klatt, M. A.; Lovrić, J.; Chen, D.; Kapfer, S. C.; Schaller, F. M.; Schönhöfer, P. W. A.; Gardiner, B. S.; Smith, A.-S.; Schröder-Turk, G. E.; Torquato, S. Universal Hidden Order in Amorphous Cellular Geometries. Nat. Commun. 2019, 10, 811.

(28) Slivina, E.; Abass, A.; Bätzner, D.; Strahm, B.; Rockstuhl, C.; Fernandez-Corbaton, I. Insights into Backscattering Suppression in Solar Cells from the Helicity-Preservation Point of View. Phys. Rev. Appl. 2019, 12, 054003.

(29) Fernandez-Corbaton, I. Forward and Backward Helicity Scattering Coefficients for Systems with Discrete Rotational Symmetry. Opt. Express 2013, 21, 29885-29893.

(30) Fernandez-Corbaton, I.; Zambrana-Puyalto, X.; Tischler, N.; Vidal, X.; Juan, M. L.; Molina-Terriza, G. Electromagnetic Duality 
Symmetry and Helicity Conservation for the Macroscopic Maxwell's Equations. Phys. Rev. Lett. 2013, 111, 060401.

(31) Bottein, T.; Wood, T.; David, T.; Claude, J. B.; Favre, L.; Berbézier, I.; Ronda, A.; Abbarchi, M.; Grosso, D. Black” Titania Coatings Composed of Sol-Gel Imprinted Mie Resonators Arrays. Adv. Funct. Mater. 2017, 27, 1604924.

(32) Verschuuren, M. A.; Megens, M.; Ni, Y.; van Sprang, H.; Polman, A. Large Area Nanoimprint by Substrate Conformal Imprint Lithography (SCIL). Adv. Opt. Technol. 2017, 6, 243-264.

(33) Visser, D.; Chen, D. Y.; Désières, Y.; Ravishankar, A. P.; Anand, S. Embossed Mie Resonator Arrays Composed of Compacted $\mathrm{TiO}_{2}$ Nanoparticles for Broadband Anti-Reflection in Solar Cells. Sci. Rep. 2020, 10, 12527.

(34) Slivina, E.; Bätzner, D.; Schmager, R.; Langenhorst, M.; Lehr, J.; Paetzold, U. W.; Lemmer, U.; Rockstuhl, C. The Annual Energy Yield of Mono- and Bifacial Silicon Heterojunction Solar Modules with High-Index Dielectric Nanodisk Arrays as Anti-Reflective and Light Trapping Structures. Opt. Express 2021, 29, 34494-34509.

(35) Piechulla, P. M.; Muehlenbein, L.; Wehrspohn, R. B.; Nanz, S.; Abass, A.; Rockstuhl, C.; Sprafke, A. Fabrication of NearlyHyperuniform Substrates by Tailored Disorder for Photonic Applications. Adv. Opt. Mater. 2018, 6, 1701272.

(36) Battiston, G. A.; Gerbasi, R.; Gregori, A.; Porchia, M.; Cattarin, S.; Rizzi, G. A. PECVD of Amorphous TiO2 Thin Films: Effect of Growth Temperature and Plasma Gas Composition. Thin Solid Films 2000, 371, 126-131.

(37) Lee, W. G.; Woo, S. I.; Kim, J. C.; Choi, S. H.; Oh, K. H. Preparation and Properties of Amorphous $\mathrm{TiO} 2$ Thin Films by Plasma Enhanced Chemical Vapor Deposition. Thin Solid Films 1994, 237, 105-111.

(38) Volk, A.-K.; Tucher, N.; Seiffe, J.; Hauser, H.; Zimmer, M.; Blasi, B.; Hofmann, M.; Rentsch, J. Honeycomb Structure on MultiCrystalline Silicon Al-BSF Solar Cell With 17.8\% Efficiency. IEEE J. Photovoltaics 2015, 5, 1027-1033.

(39) Piechulla, P.; Seiffe, J.; Hofmann, M.; Rentsch, J.; Preu, R. Increased Ion Energies for Texturing in a High-Throughput Plasma Tool. Proc. of 26th European Photovoltaic Solar Energy Conference and Exhibition, EUPVSEC, 2011; DOI: 10.4229/26thEUPVSEC20112CV.2.16.

(40) Gueymard, C. A.; Myers, D.; Emery, K. Proposed Reference Irradiance Spectra for Solar Energy Systems Testing. Sol. Energy 2002, $73,443-467$.

(41) Torquato, S.; Uche, O. U.; Stillinger, F. H. Random Sequential Addition of Hard Spheres in High Euclidean Dimensions. Phys. Rev. E 2006, 74, 061308.

(42) Yu, S.; Qiu, C.-W.; Chong, Y.; Torquato, S.; Park, N. Engineered Disorder in Photonics. Nat. Rev. Mater. 2021, 6, 226-243.

(43) Meiners, B.-M.; Holinski, S.; Schäfer, P.; Hohage, S.; Borchert, D. Investigation of anti-reflection-coating stacks for silicon heterojunction solar cells. Proc. of 29th European Photovoltaic Solar Energy Conference and Exhibition, EUPVSEC, 2014; pp 1020-1023, DOI: 10.4229/EUPVSEC20142014-2AV.3.22.

(44) McIntosh, K. R.; Baker-Finch, S. C. OPAL 2: Rapid Optical Simulation of Silicon Solar Cells. 2012 38th IEEE Photovoltaic Specialists Conference, Austin, TX, U.S.A., June 3-8, 2012, IEEE, 2012; pp 000265-000271, DOI: 10.1109/PVSC.2012.6317616.

(45) Bassi, N.; Clerc, C.; Pelet, Y.; Hiller, J.; Fakhfouri, V.; Droz, C.; Despeisse, M.; Levrat, J.; Faes, A.; Bätzner, D. GridTouch: Innovative Solution for Accurate IV Measurement of Busbarless Cells in Production and Laboratory Environments. Proc. of 29th European Photovoltaic Solar Energy Conference and Exhibition, EUPVSEC, 2014; p 1180, DOI: 10.4229/EUPVSEC20142014-2BV.8.24.

(46) Pomplun, J.; Burger, S.; Zschiedrich, L.; Schmidt, F. Adaptive Finite Element Method for Simulation of Optical Nano Structures. Phys. Status Solidi B 2007, 244, 3419-3434.

(47) Schinke, C.; Christian Peest, P.; Schmidt, J.; Brendel, R.; Bothe, K.; Vogt, M. R.; Kröger, I.; Winter, S.; Schirmacher, A.; Lim, S.; Nguyen, H. T.; MacDonald, D. Uncertainty analysis for the coefficient of band-to-band absorption of crystalline silicon. AIP Adv. 2015, 5, 067168.

(48) Holman, Z. C.; Filipič, M.; Descoeudres, A.; De Wolf, S.; Smole, F.; Topič, M.; Ballif, C. Infrared Light Management in HighEfficiency Silicon Heterojunction and Rear-Passivated Solar Cells. J. Appl. Phys. 2013, 113, 013107.

(49) Jellison, G. E.; Modine, F. A. Parameterization of the Optical Functions of Amorphous Materials in the Interband Region. Appl. Phys. Lett. 1996, 69, 371-373.

(50) Oskooi, A. F.; Roundy, D.; Ibanescu, M.; Bermel, P.; Joannopoulos, J. D.; Johnson, S. G. Meep: A flexible free-software package for electromagnetic simulations by the FDTD method. Comput. Phys. Commun. 2010, 181, 687-702.

(51) Uche, O. U.; Stillinger, F. H.; Torquato, S. Constraints on Collective Density Variables: Two Dimensions. Phys. Rev. E 2004, 70, 046122.

(52) Born, M.; Wolf, E.; Bhatia, A. B.; Clemmow, P. C.; Gabor, D.; Stokes, A. R.; Taylor, A. M.; Wayman, P. A.; Wilcock, W. L. Principles of Optics: Electromagnetic Theory of Propagation, Interference and Diffraction of Light, 7th ed.; Cambridge University Press, 1999.

(53) Keller, J. Stochastic Equations and Wave Propagation in Random Media. Proc. Symp. Appl. Math. 1964, 16, 145-170.

(54) Mandel, L.; Wolf, E. Optical Coherence and Quantum Optics; Cambridge University Press: Cambridge, U.S.A., 1995. 